\title{
Half pipe snowboarding: an (un)forgettable experience or an increasing risk for head injury?
}

\section{G P Rajan, R Zellweger}

Br J Sports Med 2004;38:e35 (http://www.bjsportmed.com/cgi/content/full/38/6/e35). doi: 10.1136/bjsm.2004.011882

The case is presented of a professional half pipe snowboarder with a large post-traumatic subdural haematoma, which allowed close to normal functioning and socialisation. It is an example of a potentially life threatening sports injury in an increasingly commercialised and popular winter sport. It highlights that a knowledge of injury patterns and a high level of suspicion should be maintained in the treatment of snowboarding injuries.

\section{CASE REPORT}

A 26 year old healthy professional snowboarder presented with headaches and difficulty in memorising numbers for about four weeks. He also complained about his recently declining short term memory. A detailed history revealed two episodes of unremarkable blunt head traumas caused by snowboard crashes during half pipe training two months previously, without obvious sequelae. On presentation, his Glasgow coma scale was 15, pupil reactions were normal, and motor strength of the right arm was slightly reduced (M4) accompanied by a right sided hyper-reflexia. The remainder of the clinical examination was unremarkable. Because of the focal neurological signs, a magnetic resonance imaging scan of the head was obtained which revealed an enormous left sided subdural haematoma with a major midline shift and compression of the left lateral ventricle and hemisphere (figs 1-3).

Cranial burr hole trepanation under local anaesthesia was performed, and the haematoma was evacuated. The postoperative course was uneventful. Follow up two weeks later showed complete resolution of the neurological, cognitive, and mnemic deficits.

\section{DISCUSSION}

Snowboarding has become the most popular winter sport during the last decade. As a consequence, related injuries have increased dramatically. ${ }^{2}$ A comparison with alpine skiing shows a four to six times higher incidence of head injuries in snowboarders, with subdural haematoma being the most common intracranial pathology. ${ }^{3}$ For this reason, knowledge of the sport specific injury patterns and a high level of suspicion should be maintained in the treatment of snowboarding injuries to minimise misdiagnosis and undertreatment. ${ }^{2}$

In general, subdural haematomas are the most common traumatic manifestation of intracranial, extra-axial haemorrhage. ${ }^{5}$ They are termed acute, subacute, or chronic, depending on the time of their first appearance and duration. Acute types occur immediately after trauma, and the subacute evolve within two months. Subdural haematomas persisting

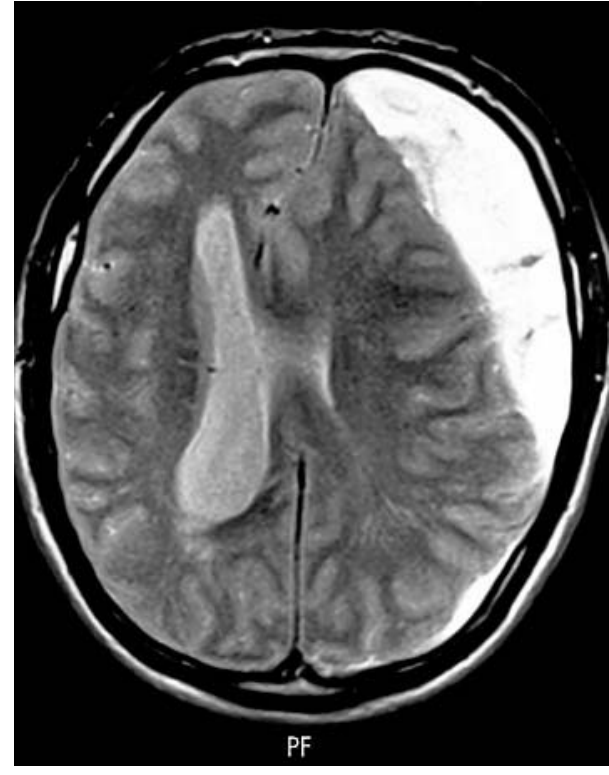

Figure 1 Axial, T2 weighted, fat suppressed magnetic resonance imaging section. Note the subdural hemispheric haematoma causing the midline shift and obliteration of the frontal recess and central part of the left lateral ventricle.

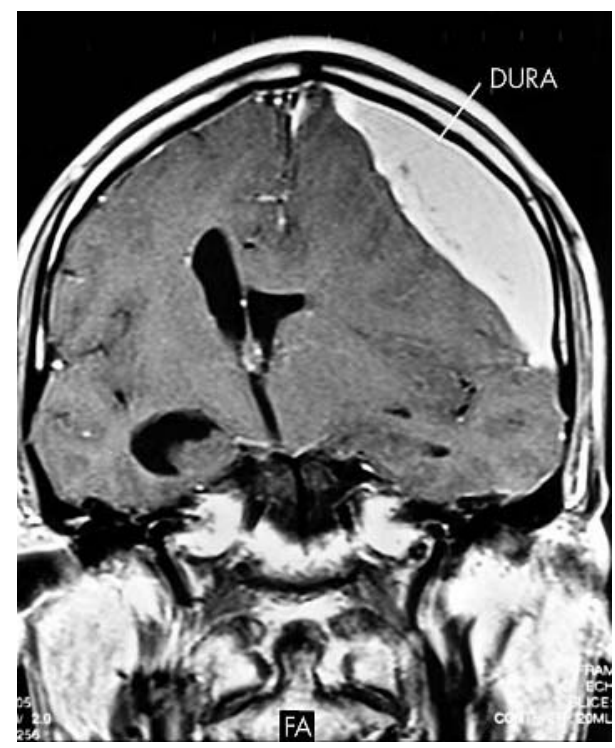

Figure 2 Coronal $\mathrm{T} 1$ weighted magnetic resonance imaging section. Note the haematoma in the subdural space between the preserved dural lining and the cerebral cortex, the midline shift and the total obliteration of the temporal horn of the left lateral ventricle. 


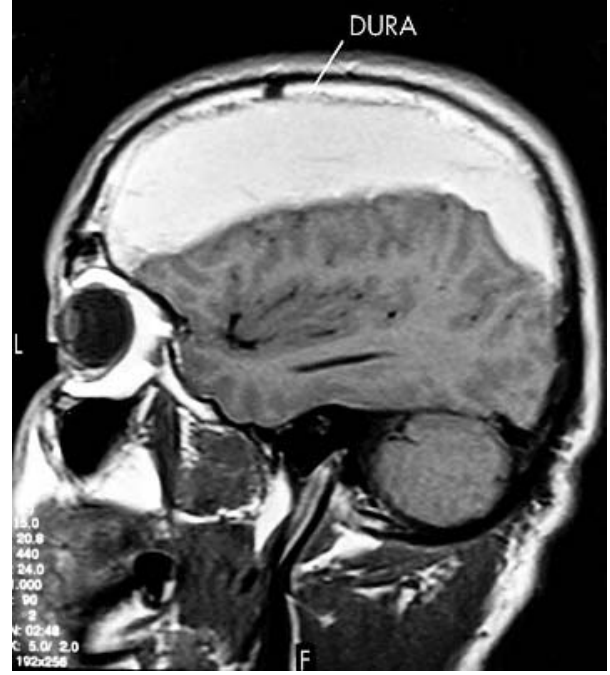

Figure 3 Sagittal Tl weighted magnetic resonance imaging section. Note the preservation of the dural lining and the extent of the hemispheric compression with effacement of the sulci and gyri due to the haematoma.

for longer than two months fall into the category of chronic. $^{56}$ The subdural bleeding results from tearing or avulsion of the bridging veins between the cortex and the dural venous sinus. As a consequence, the haematoma occupies the subdural space, thereby compressing the neighbouring brain. These effects are responsible for the typical crescent appearance of a subdural haematoma in diagnostic imaging of the neurocranium, contrasting the biconvex, lenticular shape of an epidural haematoma. ${ }^{56}$

The subsequent rapid intracranial mass effect leads to progressive neurological deterioration, which is a life threatening surgical emergency. ${ }^{5}$ Slow, gradual expansion is compensated for by the central nervous system, displaying the exceptional plasticity of the brain. These mechanisms are responsible for the asymptomatic course in chronic cases, which can last for several years. ${ }^{5-7}$ Any mnemic, cognitive, or focal deficits occurring after a head injury must dictate further investigation to rule out subdural haemorrhage or other intracranial pathology. Treatment consists of burr hole trepanation and haematoma evacuation, rarely requiring additional haemostatic measures. The long term prognosis has proved to be excellent.. ${ }^{78}$
What is already known on this topic

A lot is known about snowboarding as a sport, but there are only a few articles on related injuries. As snowboarding has become the most popular winter sport in Europe and North America, awareness about the injury risks of snowboarders must be increased.

\section{What this study adds}

This case report is intended to alert physicians in order to minimise undertreatment in this patient group. It shows that history taking and physical examination are crucial to the outcome. As snowboard professionals are used to having falls without hardly noticing, it is the challenge of the medical profession to exclude/find injuries using a knowledge of injury patterns. This report adds to our knowledge of head injuries in snowboarders.

\section{Authors' affiliations}

G P Rajan, Sir Charles Gairdner Hospital, Nedlands, Perth, WA, Australia

R Zellweger, Groote Schuur Hospital, Cape Town, South Africa

Correspondence to: Dr Rajan, Sir Charles Gairdner Hospital, Otolaryngology, Head and Neck Surgery, Verdun Street, Nedlands, WA 6009, Australia; guneshrajan@hotmail.com

Accepted 16 March 2004

\section{REFERENCES}

1 Abu-Laban RB. Snowboarding injuries: an analysis and comparison with alpine skiing injuries. CMAJ 1991;145:1097-103.

2 Bladin C, McCrory P. Snowboarding injuries: nn overview. Sports Med 1995; 19:358-64.

3 Nakaguchi H, Fujimaki T, Ueki K, et al. Snowboard head injury: prospective study in Chino, Nagano, for two seasons from 1995 to 1997. J Trauma 1999;46:1066-9.

4 Fukuda $\mathrm{O}$. Head injuries in snowboarders compared with head injuries in skiers. A prospective analysis of 1076 patients from 1994 to 1999 in Niigata, Japan. Am Sports Med $2001 ; 29: 437-40$

5 Drapkin AJ. Chronic subdural haematoma: pathophysiological basis for treatment. Br J Neurosurg 1991;5:467-73.

6 Laidlow DJ. Chronic subdural haematoma. In: Kaye AH, Black PMcL, eds. Operative neurosurgery. Churchill Livingstone, 2000;1:241-50.

7 Sambasivan M. An overview of chronic subdural haematoma: experience with 2300 cases. Surg Neurol 1997;47:418-22.

8 Uzura M, Taguchi Y, Matsuzawa M, et al. Chronic subdural haematoma after snowboard head injury. Br J Sports Med 2003;37:82-3. 